\title{
Clinical Profile and Outcome of Patients with Dengue Syndrome In Hospital Care
}

\author{
HANI F MOHAMMAD ${ }^{1}$, DEVENDRA NATH SARKAR ${ }^{2}$, M ROBED AMI N $^{3}$, A BASHER $^{4}$, T AHMED $^{5}$
}

\begin{abstract}
:
Dengue is a viral febrile illness, which has become a major international public health concern including Bangladesh, spectrum of illness ranging from dengue fever (DF) to life threatening dengue haemorrhagic fever (DHF), dengue shock syndrome (DSS) and ultimately death. The clinical profiling and outcome during epidemic dengue outbreak was observed in Dhaka Medical College Hospital in 2000 to 200I. One hundred and fifty patients were systematically observed. Male female ratio was 5:I with severity of illness in younger patients. Hundred percent patients had fever with $83 \%$ having generalized weakness. The severe cases(DHF) were having sudden sustained temperature of 103 degree farenheight. Majority have abdominal symptoms (>80\%) while 10\% had respiratory only. Sixty percent have hepatomegaly while $40 \%$ spleenomealy while $80 \%$ of DHF had ascites. Above $75 \%$ cases of Dengue haemorhagic fever had positive tourniquet test while melaena was the commonest mucosal bleeds. No fatality was observed in this study although variability of discharge was seen in different groups.
\end{abstract}

Keyword: Clinical, Profile, Outcome, Dengue, Hospital

\section{Introduction:}

Dengue fever pronounced as "Dhen Gey" is a febrile viral illness transmitted by arthropod vectors. ${ }^{1}$

Since its first recognition during the last quarter of $18^{\text {th }}$ century, periodic outbreak has been reported from both the developed and developing countries with Asia always remaining the area of highest endemicity. ${ }^{2,3}$ In 1964 there was an outbreak of dengue and chikungunya virus infection called "Dacca fever" which was the $1^{\text {st. }}$ documented outbreak of dengue in Bangladesh. ${ }^{4}$ Another epidemic fever with features closely mimicking that of dengue haemorrhagic fever occurred again in 1968 in areas of Bangladesh bordering Myanmar. ${ }^{5}$ The associated severe haemorrhagic manifestations took a heavy toll during the outbreak. Dengue has not been a public health problem before July 2000 outbreak, so there was little evidence and awareness in this regard. From hospital record of Dhaka Medical College Hospital it is seen that 1087 dengue subjects were admitted in Dhaka Medical College Hospital from July 2000 to $31^{\text {st }}$ December 2000, in this 6(six) months of which 15 patient died. It is likely that the dengue epidemic will continue to increase in frequency and magnitude in future. Bangladesh is a country where most infectious diseases are prevailing in endemic level through mosquitoes and dengue is a similar mosquito vector borne disease. ${ }^{1}$ All the favourable environments and other related factors are not only present but also developing rapidly further providing an ever-expanding potential field for the dengue endemicity and epidemics. There is no facility in our country to diagnose most of the viral infections specifically by laboratory investigations like virus isolation by culture, so we are lacking the experience of these diseases in detail. Therefore, evaluation of clinical features and management outcome of the subjects with dengue fever is essential for the development of uniform diagnostic criteria, management approach and to identify the risk groups of patients to curtail the morbidity and mortality of this disease.

In this study we tried to determine the risk group of patients suffering from dengue syndrome so as to cared and managed promptly, to determine the clinical parameters of the subjects hospitalized for dengue fever, to find out the pattern of presentation of dengue fever in hospital care and to put forward an overview about dengue fever in Bangladesh to the general public, health care providers and professionals.

1. Assistant Professor of Medicine, Sir Salimullah Medical College

2. Junior Consultant of Medicine, Sir Salimullah Medical College

3. Assistant Professor of Medicine, Dhaka Medical College

4. Medical Officer, Kashba Health Complex, Brammonbaria

5. Professor of Medicine (Rtd)

Correspondence : Dr .Md. Robed Amin, Assistant Professor of Medicine, Department of Medicine, Dhaka Medical College, Bangladesh.Apt-C-2, House no-76, Road-5, Block-F, Banani, Dhaka, Bangladesh. e.mail : robedamin@yahoo.com 


\section{Materials and Methods:}

It is a prospective observational study carried out in 150 patients despite age and sex variation admitted in different medicine and paediatrics units of Dhaka Medical College Hospital from July 2000 to March 2001. Detailed clinical history and physical examination data was recorded for each individual patient in a predesigned case-record form (CRF).

Study subjects (patients) are classified into 03(Three) groups depending on the clinical and laboratory findings. Group I (DF) is subjects with dengue fever, Group 2 (DHF) is Subjects with dengue haemorrhagic fever and Group 3 (DSS) is Subjects with dengue shock syndrome. The group 1 is selected from Subjects with acute febrile illness for not more than 2-7 days having the manifestations like-Sudden onset of fever plus Two or more of the following features:

I. Severe headache.

II. Retro orbital pain

III. Severe myalgia, arthralgia, backache

IV. Leukopenia plus

$\mathrm{V}$. Absence of convincing evidence of any other febrile illness

Group II are Subjects with dengue fever at the initial stage plus haemorrhagic manifestations evidenced by one or more of the followings.

I. Postive tourniquet test.

II. Petechiae, Ecchymosis, purpura,

III. Bleeding from different sites like epistaxis, gum bleeding, haematemesis, melaena, haematuria, menorrhagia and

IV. Any evidence of plasma leakage manifested by

a. A $\geq 20 \%$ rise in Hct for the age or sex and/or

b. A $\geq 20 \%$ drop in Hct following treatment with intravenous fluid and/or

c. Pleural effusion /Ascites / hypoproteinemia.

Group III are subjects with Dengue haemorrhagic fever manifesting circulatory failure as follows

I. Hypotension for age and / or

II. Narrow pulse pressure $(<20 \mathrm{mmHg})$ and / or Profound shock

At the initial phase these groupings could not be ensured . The course of the disease is a continuum passing from one group to the another and the transient period is during the afebrile phase. Hence the above mentioned grouping was the ultimate classification in the course of time. Pregnancy, patients suspected of suffering from any other febrile illness and revealing on the laboratory investigations and patients suffering from other chronic ailments in addition to dengue fever such as chronic renal failure, diabetes mellitus, skin infections, immunocompromised or haematological disorders were excluded from the study.

SAMPLE SIZE: 150 subjects on the above criteria was taken for the study as random fashion. Purposive convenient sample was taken. Enrolled patients were seen by the investigators clinically and follow up in hospital until discharge or fatality. Informed written consent was taken from patients or their attendant before enrolment and after discussing the protocol. A prestructured questionnaire were filled up by the investigators after a clinical diagnosis of dengue syndrome and later the groups were encoded after the temporal profile of disease were clear cut. After enrolment $5 \mathrm{ml}$ blood was taken on admission day for Complete Blood Count including - TC, DC of WBC ESR, Hb\%, Malarial Parasite count, Widal test, Urine for routine microscopic examination, Serum ALT, AST, bilirubin, CXR, USG of abdomen, Blood urea, creatinine, Serum electrolytes. Platelet count 12 hourly every day and Haematocrit on every day was done in every patients. Antibody IgM, IgG for dengue were done after 7 days of fever. Few patients of jaundice, serological tests were also done to exclude other hepatrotropic virus infection.

Serotype identification and virus isolation could not be done due to lack of facilities.

All data were analyzed by SPSS version 10 IllinoisChicago,USA and expressed in tabulated form. The study was approved by ethical review committee of the institution of Dhaka Medical College and in every step of data collection and patients care, ethical consideration was observed critically.

\section{Results:}

A total of 150 patients suffering from dengue fever were included in this study, of them $84.67 \%$ (127) belonged to group-II i.e dengue haemorrhagic fever, $12 \%$ (18) belonged to group-1 i.e dengue fever and 3.33\% (5) belonged to groupIII i.e dengue shock syndrome. Majority of study subjects were male and male to female ratio is 5:1.

As regards to the age, group III patients of dengue shock syndrome cases were significantly younger as compared to the other two groups \& $100 \%$ are below 20 years of age. Group-II i.e dengue haemorrhagic fever cases were slightly older as compared to other groups but not statistically significant. Mean age of group-II subjects were 27.59 1.18 and $26.75 \pm 3.69$ years for group- 1 subjects.

Demographic pictures of three groups of patients with comparison of age, hospital stay, temp shown in table 1 . 
All the study subjects had fever \& in almost all of them onset of fever was sudden. 100\% of group-I \& group-III and 93.77\% of group-II subjects had sudden onset of fever. Only a small percentage of subjects i.e $22.2 \%, 30.7 \%$ \& $60 \%$ of group-I, group-II and group-III respectively experienced chills and rigors. Majority of the subjects (83\%) including all three groups experienced severe weakness.

About $72.2 \%$ of group-I, $100 \%$ of group-III and $46.2 \%$ of group-II subjects suffered from temperature above $103^{0} \mathrm{~F}$ while mean temperature of group-I, group-II and group-III were $103.45 \pm 0.28,103.08 \pm 0.13$ and $104.00 \pm 1.00$ degrees Fahrenheit respectively. Sustained temperature was experienced by $100 \%, 44.4 \%$ and $26 \%$ subjects of group-III, group-I and group-II respectively. While intermittent fever was recorded in 54.3\%, $44.4 \%$ and $0.00 \%$ subjects of groupII, group-I and group-III, biphasic temperature was observed in only $18.1 \%$ (23), $11.1 \%$ (2) and $0.00 \%$ subjects of group-II, group-I and group-III as shown in Table-II.

Mean duration of fever were found $5.50 \pm 0.51$ days in groupI, 6.12 \pm 0.2 days in group-II and 5.00 \pm 1.15 days in group-III subjects. Twenty patients in group II were seen to have fever more than usual 7 days and one patient had 14 days of fever. Majority subjects i.e more than $77 \%$ of all groups experienced different grades of sweating predominantly of moderate grade of sweating.

Almost all the subjects of each group suffered from headache (more than 78\%) and myalgia (more than 81\%).Hundred percent of group-III subjects had the complain of headache, myalgia, low-back pain, retro-orbital pain and arthralgia while retroorbital pain was experienced by one third of group-I (33.3\%) and group-II (33.9\%) subjects. Low back pain was recorded in $44.4 \%$ of group-I and $53.5 \%$ of group-III subjects (Table-III).

Table-I

Demographic parameters and comparison between groups of study subjects.

\begin{tabular}{lccccc}
\hline Groups & $\begin{array}{c}\text { Agein years } \\
\mathrm{M} \pm \mathrm{SEM}\end{array}$ & $\begin{array}{c}\text { Hosp Stayin days } \\
\mathrm{M} \pm \mathrm{SEM}\end{array}$ & $\begin{array}{c}\text { Duration of feverin days } \\
\mathrm{M} \pm \mathrm{SEM}\end{array}$ & $\begin{array}{c}\text { Highest Tempin } \\
{ }^{\circ} \mathrm{F}\end{array}$ & $\begin{array}{c}\text { Cured in days } \\
\mathrm{M} \pm \mathrm{SEM}\end{array}$ \\
\hline Group - I N = 18 & $26.75 \pm 3.69$ & $5.17 \pm 0.74$ & $05.50 \pm 0.51$ & $103.45 \pm 0.28$ & $9.60 \pm 1.21$ \\
Group - II N = 127 & $27.59 \pm 1.18$ & $05.82 \pm 0.36$ & $6.12 \pm 0.21$ & $103.08 \pm 0.13$ & $11.22 \pm 0.41$ \\
Group - III N = 5 & $10.67 \pm 2.33$ & $10.67 \pm 1.20$ & $05.00 \pm 1.15$ & $104.00 \pm 1.00$ & $20.00 \pm 4.00$ \\
\hline
\end{tabular}

Table-II

Different pattern of fever in study patients according to groups

\begin{tabular}{lcccccc}
\hline Pattern of fever & \multicolumn{2}{c}{ Group-I $(\mathrm{n}=18)$} & & \multicolumn{2}{c}{ Group-II $(\mathrm{n}=127)$} & \multicolumn{2}{c}{ Group-III $(\mathrm{n}=05)$} \\
\cline { 2 - 7 } \cline { 5 - 7 } & No. & $\%$ & & No. & $\%$ & \\
\hline Sustained & 08 & 44.4 & 33 & 26.0 & 05 & 100 \\
Intermittent & 08 & 44.4 & 69 & 54.3 & 00 & 00 \\
Remittent & 00 & 00 & 00 & 00 & 00 & 00 \\
Relapsing & 00 & 00 & 02 & 01.6 & 00 & 00 \\
Biphasic & 02 & 11.1 & 23 & 18.1 & 00 & 00 \\
\hline
\end{tabular}

Table-III

Distribution of the study subjects according to pain pattern experienced by them.

\begin{tabular}{|c|c|c|c|c|c|c|}
\hline \multirow[t]{2}{*}{ Pain Pattern } & \multicolumn{2}{|c|}{ Group-I $(\mathrm{n}=18)$} & \multicolumn{2}{|c|}{ Group-II (n=127) } & \multicolumn{2}{|c|}{ Group-III (n=05) } \\
\hline & No. & $\%$ & No. & $\%$ & No. & $\%$ \\
\hline Headache & 17 & 94.4 & 100 & 78.7 & 5 & 100 \\
\hline Myalgia & 17 & 94.4 & 104 & 81.9 & 5 & 100 \\
\hline Low back pain & 08 & 44.4 & 68 & 53.5 & 5 & 100 \\
\hline Retro-orbital pain & 06 & 33.3 & 43 & 33.9 & 5 & 100 \\
\hline Arthralgia & 05 & 27.8 & 56 & 44.1 & 5 & 100 \\
\hline Arthritis & 00 & 00 & 22 & 17.6 & 00 & 00 \\
\hline
\end{tabular}


None of the study subjects has suffered from shortness of breathing but only a small number of subjects of group-I and group-II complained of cough, chest pain and prostration. Anorexia, nausea and vomiting were the common complains of the study subjects. Although diarrhoea and abdominal pain were infrequent complains of group-I and group-II subjects. But $100 \%$ subjects of group-III has suffered from these two complains like diarrhoea and abdominal pain. (Table-IV)
Organomegaly was observed very rarely except in group-III where $60 \%$ subjects had enlarged liver and $40 \%$ subjects had enlarged spleen. Majority of this group-III subjects had ascites (80\%) and 40\% had pleural effusion. No patient had lymphadenopathy in this 150 patients (table-V).

Different pattern of rash over the trunk i.e macular, maculopapular and erythematous rash were observed in the study subjects. $67 \%$ of group-I and $60 \%$ of group-II and group-III subjects had no rash (table-VI).

Table-IV

Respiratory and abdominal symptoms in three groups of patients

\begin{tabular}{|c|c|c|c|c|c|c|}
\hline \multirow[t]{2}{*}{ Symptoms PresentRespiratory } & \multicolumn{2}{|c|}{ Group-I $(n=18)$} & \multicolumn{2}{|c|}{ Group-II $(n=127)$} & \multicolumn{2}{|c|}{ Group-III $(n=05)$} \\
\hline & No. & $\%$ & No. & $\%$ & No. & $\%$ \\
\hline Shortness of breath & 00 & 00 & 00 & 00 & 00 & 00 \\
\hline Cough & 02 & 11.1 & 22 & 17.3 & 00 & 00 \\
\hline Chest pain & 02 & 11.1 & 33 & 26.0 & 00 & 00 \\
\hline Prostration & 02 & 11.1 & 16 & 12.6 & 00 & 00 \\
\hline \multicolumn{7}{|l|}{ Abdominal symptoms } \\
\hline Anorexia & 15 & 83.3 & 77 & 60.6 & 5 & 100 \\
\hline Nausea & 17 & 94.4 & 75 & 59.1 & 5 & 100 \\
\hline Vomiting & 13 & 72.2 & 92 & 74.4 & 5 & 100 \\
\hline Diarrhoea & 03 & 16.7 & 39 & 30.7 & 5 & 100 \\
\hline Abdominal distension & 00 & 00 & 08 & 06.3 & 00 & 00 \\
\hline Abdominal pain & 06 & 33.3 & 35 & 27.6 & 5 & 100 \\
\hline
\end{tabular}

Table-V

Clinical signs observed in three groups of dengue syndrome

\begin{tabular}{|c|c|c|c|c|c|c|}
\hline \multirow[t]{2}{*}{ Organomegaly \& ascites/effusion. } & \multicolumn{2}{|c|}{ Group-I $(\mathrm{n}=18)$} & \multicolumn{2}{|c|}{ Group-II (n=127) } & \multicolumn{2}{|c|}{ Group-III (n=05) } \\
\hline & No. & $\%$ & No. & $\%$ & No. & $\%$ \\
\hline Spleen & 00 & 00 & 02 & 1.6 & 2 & 40 \\
\hline Liver & 00 & 00 & 10 & 7.9 & 3 & 60 \\
\hline L. Node & 00 & 00 & 00 & 00 & 00 & 00 \\
\hline Spleen \& liver & 00 & 00 & 00 & 00 & 00 & 00 \\
\hline Ascites & 00 & 00 & 02 & 1.6 & 04 & 80 \\
\hline Pl. Effusion & 00 & 00 & 02 & 1.6 & 02 & 40 \\
\hline Ascites \& Effusion & 00 & 00 & 04 & 3.1 & 02 & 40 \\
\hline
\end{tabular}

Table-VI

Rashes of different types observed in different groups of patients

\begin{tabular}{|c|c|c|c|c|c|c|}
\hline \multirow[t]{2}{*}{ Types of Rash } & \multicolumn{2}{|c|}{ Group-I $(n=18)$} & \multicolumn{2}{|c|}{ Group-II $(n=127)$} & \multicolumn{2}{|c|}{ Group-III $(n=05)$} \\
\hline & No. & $\%$ & No. & $\%$ & No. & $\%$ \\
\hline Macular & 2 & 11.1 & 14 & 11.0 & 2 & 40 \\
\hline Maculo-papular & 2 & 11.1 & 13 & 10.2 & 00 & 00 \\
\hline Erythematous & 2 & 11.1 & 23 & 18.1 & 00 & 00 \\
\hline None & 12 & 66.7 & 77 & 60.6 & 3 & 60 \\
\hline
\end{tabular}


Table-VII

Haemorhagic manifestation of variable groups of dengue patients.

\begin{tabular}{|c|c|c|c|c|c|c|}
\hline \multirow{2}{*}{$\begin{array}{l}\text { Diff. types of haemorrhagic } \\
\text { manifestations }\end{array}$} & \multicolumn{2}{|c|}{ Group-I $(\mathrm{n}=18)$} & \multicolumn{2}{|c|}{ Group-II (n=127) } & \multicolumn{2}{|c|}{ Group-III (n=05) } \\
\hline & No. & $\%$ & No. & $\%$ & No. & $\%$ \\
\hline +Ve Tourniquet. Test & 03 & 16.7 & 95 & 74.8 & 04 & 80 \\
\hline Melaena & 00 & 00 & 83 & 65.3 & 05 & 100 \\
\hline Gum bleeding & 00 & 00 & 47 & 37.0 & 05 & 100 \\
\hline Haematuria & 00 & 00 & 06 & 04.7 & 00 & 00 \\
\hline Conj. hge. & 00 & 00 & 27 & 21.3 & 01 & 20 \\
\hline Petechial hge & 00 & 00 & 06 & 04.7 & 00 & 00 \\
\hline Ecchymosis. & 00 & 00 & 12 & 09.4 & 00 & 00 \\
\hline Purpura & 00 & 00 & 15 & 11.8 & 00 & 00 \\
\hline Haematemesis & 00 & 00 & 31 & 24.40 & 00 & 00 \\
\hline Haemoptysis & 00 & 00 & 12 & 09.4 & 00 & 00 \\
\hline Epistaxis & 00 & 00 & 10 & 07.9 & 00 & 00 \\
\hline More than one manifestations. & 00 & 00 & 87 & 68.5 & 05 & 100 \\
\hline
\end{tabular}

Table-VIII

Time of cure in different groups of dengue patients.

\begin{tabular}{|c|c|c|c|c|c|c|}
\hline \multirow{2}{*}{ Time of cure of Ailment } & \multicolumn{2}{|c|}{ Group-I (n=18) } & \multicolumn{2}{|c|}{ Group-II (n=127) } & \multicolumn{2}{|c|}{ Group-III $(\mathrm{n}=05)$} \\
\hline & No. & $\%$ & No. & $\%$ & No. & $\%$ \\
\hline 01-05 days & 02 & 11.1 & 00 & 00 & 00 & 00 \\
\hline 06-08 days & 09 & 50.0 & 13 & 10.2 & 00 & 00 \\
\hline 09-11 days & 06 & 33.3 & 51 & 40.2 & 00 & 00 \\
\hline 12-14 days & 01 & 05.6 & 52 & 40.9 & 00 & 00 \\
\hline 15-17 days & 00 & 00 & 07 & 5.5 & 02 & 40 \\
\hline $18-20$ & 00 & 00 & 04 & 03.1 & 01 & 20 \\
\hline 21 days \& above & 00 & 00 & 00 & 00 & 02 & 40 \\
\hline
\end{tabular}

Melaena was the common haemorrhagic manifestations. $66.3 \%$ in group-II and $100 \%$ of group-III subjects had melaena followed by gum bleeding in $37 \%$ of group-II and $100 \%$ of group-I subjects.

More than one variety of haemorrhagic manifestations was observed in most of the patients. It is found that $68.5 \%$ of group-II and $100 \%$ of group-III subjects had multiple types of haemorrhagic manifestations. Tourniquet test was positive in $16.7 \%$ of group-I, $74.8 \%$ of group-II and $80 \%$ of group-III subjects. (Table-VII).

Unusual manifestations were observed in none of the subjects except which was seen only in $1.57 \%$ of group-II and $20 \%$ of group-III subjects. $94 \%$ of group-I and group-II and only $20 \%$ group-III subjects were discharged from hospital within 9 days. Group-III subjects stayed significantly more days in hospital than group-I and groupII subjects. All the subjects of group-I and $91.3 \%$ of groupII subjects were completely cured of disease within 14 days from onset of the disease. But group-III subjects started to be cured after 14 days and only $60 \%$ were cured after 20 days, remaining $40 \%$ took more than 20 days to be cured.(Table-VIII).

\section{Discussion:}

A total number of one hundred and fifty (150) patients suffering from Dengue syndrome were included in this study of which 18 (12\%) belonged the group-I (DF), 127 (84.67\%) group-II (DHF) and 5 (3.33\%) were in group-III (DSS) with male female ratio of 5:1. 
As regards to the age - group-III (DSS) patients were significantly $(\mathrm{p}<0.05)$ younger. Kabra Sk et al. and Ibrahim $\mathrm{NM}$ et al. in their study showed dengue shock syndrome to occur more in younger age groups, and when compared to other groups of the study $100 \%$ were below 20 years of age. 6,7 Chareonsook ' $\mathrm{O}$ et al. showed-the age of highest incidence has increased and adults are now also being affected with dengue haemorrhagic fever/dengue shock syndrome. ${ }^{8}$ Wali JP et al. showed that contrary to the popular belief dengue haemorrhagic fever/dengue shock syndrome is not a disease of children only and the mean age of his study was 31 yrs \pm 5.2 (SD). ${ }^{9}$ Most of the patients were in the age range of 20-50 years, which is almost similar to this study.

All the study subjects had fever and almost in all of them onset of fever was sudden. Only a small number of subjects i.e $22.2 \%, 30.7 \%$ and $60 \%$ of group I, group-II and group-III respectively experienced chills and rigor. Anuradha $\mathrm{S}$ et al. found in her study that the fever was of high grade associated with chills and rigors and was self limiting. ${ }^{10}$ Majority of patients (>88\%) of all groups experienced severe weakness. Rigau JG et al. found that febrile period leaves the patient feeling severely tired for several more days. ${ }^{1}$ Majority of patients ( $>77 \%)$ of all groups experienced different grades of sweating predominantly of moderate grade which are consistent with other studies. Sustained temperature was experienced by $100 \%, 44 \%$ and $36 \%$ of group-III, groupI and group II subjects respectively while intermittent fever was recorded in $54.3 \%, 44.4 \%$ in group-II and group-I respectively and in none of group-III subjects. Saddleback or biphasic temperature pattern was observed in 18.1\% (23) in Group-II, $11.1 \%$ in group-I and in none of group-III subjects though biphasic or saddleback temperature curve is not the norm. Mean duration of fever were $5.50 \pm 0.51$ days, $6.12 \pm 0.02$ days and $5.00 \pm 1.15$ days in group-I, group-II and group-III respectively. Anuradha $\mathrm{S}$ et al. also reported similar average duration of about $4.8 \pm 1.32$ days. Wali JP et al. reported that fever was between $3-6$ days, mean $4 \pm 1$.2 (SD) in $83.9 \%$ of his patients studied. ${ }^{9,10}$

Different pattern of pains and aches were experienced by the study subjects. Almost all the patients of each group suffered from headache (>78\%), myalgia (>81\%), arthritis (44\%), retro-orbital pain (36\%) and low back pain. Anuradha S et al. reported $96 \%$ of her study subjects suffered myalgia and other aches while Wali. JP et al. showed Headache in $80.9 \%$, myalgia $76.2 \%$, arthalgia $52.3 \%$ and these were the common symptoms which are similar to the findings in this study. Richards AL et al. reported headache in $96.7 \%$, back pain in $39.1 \%$, retro-orbital pain in $13.1 \%$ of all his 72 study subjects, which are closely related to the present study. ${ }^{9-11}$
None of the study subjects suffered from shortness of breath. Only a small number of group-I and group-II subjects complained of cough (18.1\%), chest pain (24\%) and prostration. Wali JP et al. reported cough in $10.9 \%$, Dyspnoea in $10.9 \%$ which is not consistent in present study and chest pain in $7.3 \%$ which is slightly lower than this study findings. ${ }^{9}$ Anorexia, nausea and vomiting were common complains of all study subjects. Although diarrhoea and abdominal pain were infrequent complains of group-I and group-II subjects, it is similar to the study of Wali JP et al. while $100 \%$ of groupIII subjects suffered from diarrhoea and abdominal pain which is a remarkable unique finding in this study. ${ }^{9}$ Organomegaly were observed very rarely except in groupIII where $60 \%$ had enlarged liver, $40 \%$ enlarged spleen but none had Hepato splenomegaly together. Anuradha S et al. reported hepatomegaly in $96 \%$ but splenomegaly in none while Wali JP et al. reported hepatomegaly in 16\%, splenomegaly in $12 \%$ of all his study subjects which is in fact far less than this study. 9,10

Majority of patients of group-III had ascites (80\%) and pleural effusion in $40 \%$. Kabra Sk et al. found ascites in $87 \%$, pleural effusion in $74 \%$ of the patients with dengue shock syndrome and $27 \%$ of patients with dengue haemorrhagic fever had ascites and pleural effusion. ${ }^{6}$ None of the group-II subjects in this study had pleural effusion or ascites. Hepatomegaly, pleural effusion and ascites were commonly associated with group-III subjects of this study and is similar to the observations made in 1981 Cuban epidemic. ${ }^{12}$ Jaundice was found in $2 \%$ of patients comparatively less than the study of Kabra Sk et al. ${ }^{6}$ None had hepatic encephalopathy or dengue encephalopathy.

Different pattern of rash over the trunk e.g macular $12 \%$, maculopapular $10 \%$ and erythematous $10 \%$ totaling $>37 \%$ of the study subjects were observed .Tourniquet test was positive in $16.7 \%$ of group-I, $74.8 \%$ in group-II and $80 \%$ in group-III subjects while Nimmannitya S et al. reported $84 \%$ tourniquet test positive in all the study subjects of dengue haemorrhagic fever/dengue shock syndrome. ${ }^{13}$ Kabra Sk et al. reported Tourniquet test positive in $40 \%$ of dengue fever patients, $62 \%$ of dengue haemorrhagic fever and $64 \%$ of dengue shock syndrome patients. ${ }^{6}$ Melaena was the commonest haemorrhagic manifestation, $65.3 \%$ in group-II and $100 \%$ in group-III followed by gum bleeding in 37\% of group-II and $100 \%$ group-III subjects . More than one variety of haemorrhagic manifestations was observed in most of the patients. $68.5 \%$ of group- 2 and $100 \%$ of group-III subjects had multiple types of haemorrhagic manifestations that is similar to the findings of Wali JP et al. and Anuradha S et al. ${ }^{9,10}$ Wali JP et al. showed $36.4 \%$ of his study subjects had 
bleeding manifestations after the patient has become afebrile usually within 1-2 days of defervescence of fever (Dengue haemorrhagic fever/Dengue shock syndrome usually develop around day 3-7 of illness at the time of defervescence which is an indication for intensified observation. 9,14,15 The observation was seen in present study compliments their findings too.

All the subjects of group-I and $91.3 \%$ of group-II subjects were completely cured of the disease within 14 days from onset of illness, but none of group-III subjects. They started to be cured of the disease after 14 days and only $60 \%$ were cured after 20 days and remaining 40\% took more than 20 days . Tai-Dy et al. reported the mean hospital stay was $4.2 \pm$ 1.5 days which is much less than that of this study. ${ }^{16}$ The cause may be that this was the epidemic in our country when we were perplexed and did not feel safe to discharge patients from hospital before patients were physically fit and mentally stable and confident of being cured. In fact when platelet count is on the rising trend and in the absence of clinical bleeding it is reasonably safe to discharge patients. And if so done, this may shorten each patients stay resulting in cost saving and more efficient use of hospital bed. Rigau Perez JG reported that the median duration of hospital stay was 5 days which is inconsistent with this study. ${ }^{16,17}$

\section{Conclusion:}

Since July 2000 Bangladesh has witnessed the spectacular emergence of Dengue. The nature of the disease, nonacquaintance of the professionals and unfamiliarity on the part of the general people turned the situation into a panic. Patients with dengue virus infection constituted a substantial number of hospital admitted patients in the city hospitals and clinics during the $2^{\text {nd }}$ half of the calendar year 2000. Patients with dengue syndrome showed varied presentation and the symptoms were non specific. So a large toll has been paid measured in number of death before we become alert and aware of it.

It this study an attempt has been made to find out the clinical features and varied presentations to put forward an overview about dengue fever in Bangladesh to create better awareness and clinically diagnostic skills among the health care providers and people to identify and refer the patients promptly to proper health care facilities. Because early recognition and prompt and aggressive management is the mainstay to avert the ultimate danger. Evaluation of clinical features of the subjects with dengue fever may thus help development of uniform diagnostic criteria and management approach.
Dengue has shown its presence in Bangladesh. So before time is elapsed we must be prepared to face the future malady efficiently and appropriately by being acquainted with it. This is a preliminary observational study. Further detailed, meticulous, multi-centered and large-scale study could shed clear light on this proposition and could clearly define the situation.

\section{Conflict of Interest : None}

\section{References:}

1. Rigau Perez JG, Clark-GG, Gubler DJ, Reiter P, Sanders EJ, Vorndam AV: Dengue and Dengue haemorrhagic fever. Lancet 1998 September, 19; 352(9132): 071-7

2. Carey DE: Chikungunya and Dengue: A case of mistaken identity? J-Hist-Med 1971; 26: 243-62

3. Gubler DJ; Dengue. In Monath TP (ed). The arboviruses: Epidemiology and Ecology. Boca Raton; CRC Press, 1998: 223-280

4. Aziz MA, Gorham JR, Gregg MB: “Dacca fever.” - An outbreak of dengue fever. Pakist J Med Res 1967; 6:83-92

5. Bang $\mathrm{YH}$ and Shah NK. Regional review of Dengue haemorrhagic fever situation and control of Aedes Aegypti in South East Asia. Dengue newsletter; 1986; 12:1-9

6. Kabra SK, Jain Y, Pandey RM, Madhulika, Singhal T, Tripathi-P, Broor-S, Seth-P, Seth-V. Dengue haemorrhagic fever in children in the 1996 Delhi epidemic. Trans R-SOCTrop Med Hyg. 1999 May-Jun; 93(3): 294-8

7. Ibrahim NM, Cheong-I. Adult dengue haemorrhagic fever at Kuala Lumpur Hospital: Retrospective study of 102 cases. Br. J-Clin Pract. 1995 Jul-Aug; 49(4): 189-91

8. Chareonsook O, Foy HM, Teeraratkul-A, Silarug-N. Changing epidemiology of dengue haemorrhagic fever in Thiland. Epidemiol-Infect 1999 Feb; 122(1): 161-6

9. Wali JP, Biswas-A, Handa_R, Agarwal-P, Wig-N, Dwivedi SN. Dengue haemorrhagic fever in adults: A prospective study of 110 cases. Trop-Doct 1999 Jan; 29(1): 27-30

10. Anuradha S, Singh-NP, Rizvi-SN, Agarwal-SK, Gur-R, Mathur-MD. The 1996 outbreak of dengue haemorrhagic fever in Delhi, India. South East Asian J Trop Med public Health, 1998 Sep; 29(3):503-6

11. Richards - AI, Bagus_R, Baso SM, Follows-GA, Tan R, Graham-RR, Sandjaja-B, Corwin AL. Punjabi-N. The first reported outbreak of dengue haemorrhagic fever in Irian Jaya, Indonesia. Am j Trop Med Hyg. 1997 Jul; 57(1): 49-55.

12. Kho LM, Sumarmo, Wulur H, Jahja E, Gubler DJ. Dengue haemorrhagic fever accompanied by encephalopathy in 
Jakarta. South East Asian J Trop Med public health 1981; 12: 83-86

13. Nimmannitya S, Halstead SB, Cohen SN, Margotta MR. Dengue and Chikungunyan virus infection in Thailand 1962 - 64. I. Observations on hospitalized patients with haemorrhagic fever. Am J Trop Med Hyg 1969; 18: 954-71

14. Pan American Health Organization. Dengue and Dengue haemorrhagic fever in the Americas: Guidelines for prevention and control. Washington DC: PAHO Sci Publ No 548; 1994 : $3-20,49-58,69-70$
15. World Health Organization. Dengue haemorrhagic fever: Diagnosis, treatment and control. Geneva; WHO 1986: 715

16. Tai DY, Chee YC, Chan KW. The natural history of dengue illness based on a study of hospitalized patients in Singapore. Singapore Med J 1999 Apr. 40(4): 238-42

17. Rigau - Perez - JG. Clinical manifestations of dengue haemorrhagic fever in Puerto Rico, 1990 - 1991. Puerto Rico Association of Epidemiologists. Rev - Panam - Salud - Publica 1997 May; 1 (5): 381- 8 